\title{
Research on the Interaction between Higher Education Resource Allocation and Real Estate Price
}

\author{
Haiming Luo \\ School of Economics, Jinan University, Guangzhou, China \\ Email: 13535549383@163.com
}

How to cite this paper: Luo, H. M. (2020). Research on the Interaction between Higher Education Resource Allocation and Real Estate Price. Open Journal of Social Sciences, 8, 58-68.

https://doi.org/10.4236/jss.2020.84005

Received: March 6, 2020

Accepted: April 6, 2020

Published: April 9, 2020

Copyright $\odot 2020$ by author(s) and Scientific Research Publishing Inc. This work is licensed under the Creative Commons Attribution International License (CC BY 4.0).

http://creativecommons.org/licenses/by/4.0/

\section{(c) (i) Open Access}

\begin{abstract}
On the one hand, based on the relevant conclusions of theoretical analysis, combined with the current situation of higher education resource allocation, a higher education resource allocation evaluation system was reasonably constructed, and a comprehensive index of higher education resource allocation was obtained through principal component analysis. It is a large city in the first and second tiers, while the allocation of higher education resources in small and medium-sized cities is low. On the other hand, a quantitative model is established. By introducing the variables of higher education resource allocation in the basic model, through regression analysis, we find that at a significance level of 5\%, the regression results show that the growth rate of higher education resource allocation is related to the growth rate of real estate prices. And the relationship is significant. Combined with the empirical analysis results of this article, maybe we can start with the allocation of higher education resources. The allocation of higher education resources in first- and second-tier cities is concentrated. The government can take effective methods to allocate higher education resources to avoid regional differences in the allocation of higher education resources. It can slow down the social pressure brought by the growth of real estate prices to a certain extent.
\end{abstract}

\section{Keywords}

Higher Education Resource Allocation, Real Estate Price, Principal Component Analysis

\section{Introduction}

\subsection{Research Background}

In the academic world, domestic real estate prices and the real estate market 
have become the focus of research by domestic and foreign scholars. Many domestic and foreign scholars have begun to deeply study the influencing factors and influencing mechanisms of China's real estate prices. On the one hand, we made a qualitative analysis of various aspects of the real estate market in theory. Based on the theory of economics, the influencing factors and mechanism of real estate prices are studied. On the other hand, in empirical research, data is collected scientifically and scientifically to establish mathematical models for research in order to find out policies that effectively control real estate prices.

From the perspective of the allocation of educational resources in China, the further intensification of reform and opening up and the continuous development of the market economy have caused imbalances in regional economic development in China. The imbalances in regional economic development have led to imbalances in educational investment in various regions. There is a gap in the attractiveness of the teaching staff and college students, which means that there are some differences in higher education resources in different regions. The development of higher education will help to form human capital, improve labor productivity, and promote economic growth. With the further implementation of the strategy of rejuvenating the country through science and education, more and more research has been conducted on the allocation of higher education resources.

This difference in the allocation of higher education resources has many similarities with the regional differences in real estate prices. However, there are relatively few studies at home and abroad that link higher education resources to real estate prices, and the analysis of the real estate market is too limited. In this paper, we will study the interactive relationship between the differences in educational resources and real estate prices in various regions of China, introduce relevant mathematical models for empirical testing, analyze and summarize the impact mechanism between the two, and then give corresponding theoretical suggestions.

\subsection{Research Status}

As the research object of real estate prices, the research and analysis of them are mostly concentrated at the level of a single city or the country, demonstrated theoretically and empirically the relationship between income gap and China's real estate prices, and argued that income gap has an important impact on the rise of real estate prices ( $\mathrm{Hu}, 2010)$, analyzed the long-term existence between the real RMB exchange rate and short-term supply capital flows and the actual real estate prices in different regions by constructing the MS-VAR model using panel data containing the actual prices of RMB and short-term working capital Nonlinear dynamic relationship (Li \& Li, 2014).

With the development of panel data models and spatial econometric models, the research on the regional interactive relationship of real estate prices has attracted the attention of scholars at home and abroad. Most of the cutting-edge research on real estate prices is based on the framework of spatial dynamic panel 
data. Proceeded from the relationship between real estate prices, local public expenditure and real estate tax burden, and conducted an empirical discussion and analysis based on panel data in China's real estate market. The data were tested for stability and co-integration and empirical tests. Accuracy and scientificity of results (Du, Huang, \& Wu, 2009) analyzed the regional interaction of real estate prices in China using the Generalized Spatial Dynamic Panel Data Model and inter-provincial panel data from the first quarter of 2002 to the third quarter of 2010 (Chen \& Wang, 2012). Used 34 large and medium-sized cities in China as samples, and used econometric models and empirical tests to study the impact of the promotion pressure of local officials and the level of financial marketization on real estate prices in China (Zhu \& Xu, 2013).

The difference in the allocation of higher education resources in China is mainly reflected in the superposition of three different levels: regional, college level, and funding structure. From the perspectives of "government, market, and university", introduced a multi-layered linear model in specific analysis and carried out scientific and reasonable empirical research on the differences in the allocation of higher education resources and their impact mechanisms (Bao \& Liu, 2011). Demonstrated in an article the interactive relationship between the allocation of educational resources and housing prices, emphasizing the impact of educational capitalization on housing prices (Zhang, Li, \& Deng, 2014).

\subsection{Research Ideas}

This article will learn the relevance theory of higher education resource allocation through the study of literature materials. It will start from the two aspects of higher education resource allocation and real estate prices. In terms of regional higher education resource allocation research analysis, it will take into account the actual situation affecting higher education resource allocation. There are many factors. Select the four total indicators and conduct a principal component analysis to obtain a comprehensive index of regional higher education resource allocation. In terms of real estate prices, the average selling price of housing in each region is taken as the research object. The average sales price has a great impact, so a first-order difference is made to the average sales price of housing in each region, and the average growth rate of housing sales in each region is obtained. Finally, the interactive relationship between higher education resource allocation and real estate prices is studied to establish a reasonable measurement. The model, the impulse response model to improve the measurement model, followed by panel data for time and space dynamic research and analysis, so as to obtain the correlation between the two, and at the end give corresponding policy recommendations and opinions.

\section{Research on Higher Education Resource Allocation}

\subsection{Selection of Evaluation Indicators}

In order to evaluate the degree of higher education resource allocation among 
regions, through careful literature learning and theoretical research, based on the principles of objective fairness, systematic comprehensiveness, and operational feasibility, this article selects education funding and general higher education for the evaluation of the degree of higher education resource allocation. There are four indicators: the number of full-time teachers, the number of students in general higher education, and the abundance of general higher education institutions.

The education expenditure index is selected from the education expenditure of each city. The education expenditure of a city or a region determines the degree of government's emphasis on education. The degree of government's emphasis on education greatly affects the degree of higher education resource allocation in the region. This means that the more expenditures on education, the higher the government's emphasis on education, and the higher the allocation of higher education resources in the region.

Teachers and students, as two main subjects of education, play a decisive role in the allocation of educational resources. This article selects the indicator of the number of full-time teachers in general higher education and the number of students in general higher education. The full-time teacher of higher education itself is a direct reflection of educational resources. The more full-time teachers in a city, the more abundant the education resources in the city; The number of students in general higher education reflects the strength of urban education resources, and college students and full-time teachers have already become customers and potential customers in the real estate market, which indirectly proves that there is a certain relationship between the allocation of higher education resources and real estate prices. There is an interactive relationship between them.

The university abundance index collects the number of ordinary institutions of higher education in 35 major cities in China, and divides ordinary institutions of higher education into three categories, namely 985 institutions, 211 institutions, and other institutions, with 3 points, 2 points and 1 point are summed to obtain the city's college abundance index.

\subsection{Research on Higher Education Resource Allocation Based on 2015 Data}

For the study of the allocation of higher education resources, considering that there is no uniform caliber for measuring per capita education resources, the quantification of per capita education resources may increase the error of the experimental results, coupled with the difficulty of obtaining data, we choose An empirical analysis of the total educational resources indicators was conducted. After our collection and simple processing, we obtained the raw data of education funding, the number of full-time teachers in ordinary higher education, the number of students in ordinary higher education, and the abundance of ordinary universities in 35 major cities in China from 2006 to 2015. Firstly, based on the 2015 data, the research on the allocation of higher education resources was conducted, and the principal component analysis method was used. 
The contribution rate of each indicator obtained by plugging into the data is shown in Table 1:

Showing in Table 1 , when the cumulative contribution rate reaches $85 \%$, we believe that these indicators have explained the original indicators well. As can be seen from Table 1, the cumulative contribution rate of the first three indicators has reached $93.4709 \%$. For a more systematic and comprehensive To analyze the degree of higher education resource allocation, all four indicators should be retained. It also tells us that the number of full-time teachers has the highest degree of influence on higher education resources, accounting for $55.9403 \%$, and the number of students, education funding, and college resource abundance have an impact on the allocation of higher education resources of $27.2386 \%$, $10.3001 \%$, and $6.5210 \%$, So we feel that these four indicators should be used as the main influencing factors, that is, the principal component value, and then calculated based on the principal component value, to obtain a comprehensive index of higher education resource allocation and a comprehensive ranking in each city.

From the city rankings, we can see that the overall index of higher education resource allocation in the first-tier cities is generally higher, and the second-tier and third-tier cities are lower in turn. Among them, we can see the higher education resources in developed cities such as Beijing, Shanghai, Guangzhou The degree of allocation is high, while the allocation of higher education resources in small and medium cities such as Yinchuan and Xining is low. From this conclusion, we can conclude that the degree of higher education resource allocation is generally higher in large first-tier cities, and the degree of higher education resource allocation is generally lower in small and medium-sized cities.

\section{Empirical Research on Interaction}

\subsection{Model Establishment}

The real estate price of a city is often affected by many factors, including total urban GDP, urban per capita disposable income, total population, unemployment rate, investment demand, etc. In this study, we assume that other factors have an impact on real estate prices. It is relatively small. Ignore the influence of these factors here, and think that the total city GDP affects the real estate price. Based on this, we start to build a general panel data measurement model, which can be expressed as:

Table 1. Contribution table of each indicator.

\begin{tabular}{ccccc}
\hline \multirow{2}{*}{ City } & Full-time teachers & $\begin{array}{c}\text { Number of } \\
\text { students }\end{array}$ & Education funding & $\begin{array}{c}\text { University resource } \\
\text { abundance }\end{array}$ \\
\cline { 2 - 5 } & $55.9403 \%$ & $27.2386 \%$ & $10.3001 \%$ & $6.5210 \%$ \\
\hline $\begin{array}{c}\text { Contribution rate } \\
\text { Cumulative } \\
\text { contribution rate }\end{array}$ & $55.9403 \%$ & $83.1789 \%$ & 93.4790 & $100 \%$ \\
\hline
\end{tabular}




$$
\mathrm{REP}_{i t}=\alpha_{i}+\beta_{1} \mathrm{GDP}_{i t}+\varepsilon_{i t}
$$

In the formula, $\mathrm{REP}_{i t}$ represents the average house sales price, $\alpha_{i}$ is a constant term, $\beta_{1}$ is a regression coefficient, $\mathrm{GDP}_{i t}$ represents the total GDP of the city, and $\varepsilon_{i t}$ is the residual term. This formula is also called a static equation, assuming the above function is Logarithms can be added, and the function can be further expressed as:

$$
\ln \mathrm{REP}_{i t}=\alpha_{i}+\beta_{1} \ln \mathrm{GDP}_{i t}+\varepsilon_{i t}
$$

Since the analysis of the total urban GDP and real estate prices based on the aggregate indicator alone cannot intuitively reflect the internal relationship between the two, a first-order difference is then made to the above formula. The interactive relationship between the growth rate of real estate prices is more convincing from theoretical research and empirical analysis, and the following theoretical basic model can be obtained:

$$
\Delta \ln \mathrm{REP}_{i t}=\alpha_{i}+\beta_{1} \Delta \ln \mathrm{GDP}_{i t}+\varepsilon_{i t}
$$

Among them, $\Delta \ln \mathrm{REP}_{i t}$ represents the real estate price growth rate, and $\Delta \ln \mathrm{GDP}_{i t}$ represents the annual GDP growth rate of the city in the first year. Based on the analysis above, we believe that higher education resource allocation has an interactive relationship with real estate, and cities with high higher education resource allocation It will attract teachers and college entrance examination graduates to report, thereby speeding up the rise in residential prices. According to the above analysis, the econometric model 2 of the growth rate of real estate prices can be expressed as:

$$
\Delta \ln \mathrm{REP}_{i t}=\alpha_{i}+\beta_{1} \Delta \ln \mathrm{GDP}_{i t}+\varphi \Delta \ln \mathrm{AERI}_{i t}+\varepsilon_{i t}
$$

Among them, $\Delta \ln$ AERI $_{i t}$ represents the growth rate of the higher education allocation index. When studying the interactive relationship between higher education resource allocation and real estate prices, we take the GDP growth rate as the control variable and choose the higher education resource allocation index growth rate and real estate price growth The rate is the main variable, and the relevance of higher education resource allocation to the growth rate of real estate prices is discussed. Based on the above econometric model, descriptive statistics are made on the main variables.

Showing in Table 2, we can get the average value and standard deviation of the growth rate of real estate prices, GDP growth rate, and higher education allocation index. We can also observe the change range of the above three variables through the median, maximum, and minimum values.

Considering that the model may have a lag, that is to say, changes in the allocation of higher education resources cannot immediately respond to real estate prices. In order to analyze the relationship between the two, here is an impulse response to higher education resource allocation and real estate prices The impulse response results are shown in the figure.

Showing in Figure 1, the middle line in the figure is the optimal estimated path of the impulse response, and the upper and lower lines are the amplitude 
Table 2. Descriptive statistics.

\begin{tabular}{cccccc}
\hline & Mean & Median & Maximum value & Minimum value & Standard deviation \\
\hline$\Delta \ln R E P$ & 0.0998 & 0.0904 & 0.4545 & -0.1948 & 0.1056 \\
$\Delta \ln$ GDP & 0.1360 & 0.1307 & 0.3149 & -0.0084 & 0.0547 \\
$\Delta \ln$ AERI & 0.0929 & 0.0833 & 0.8933 & -0.8203 & 0.1070 \\
\hline
\end{tabular}

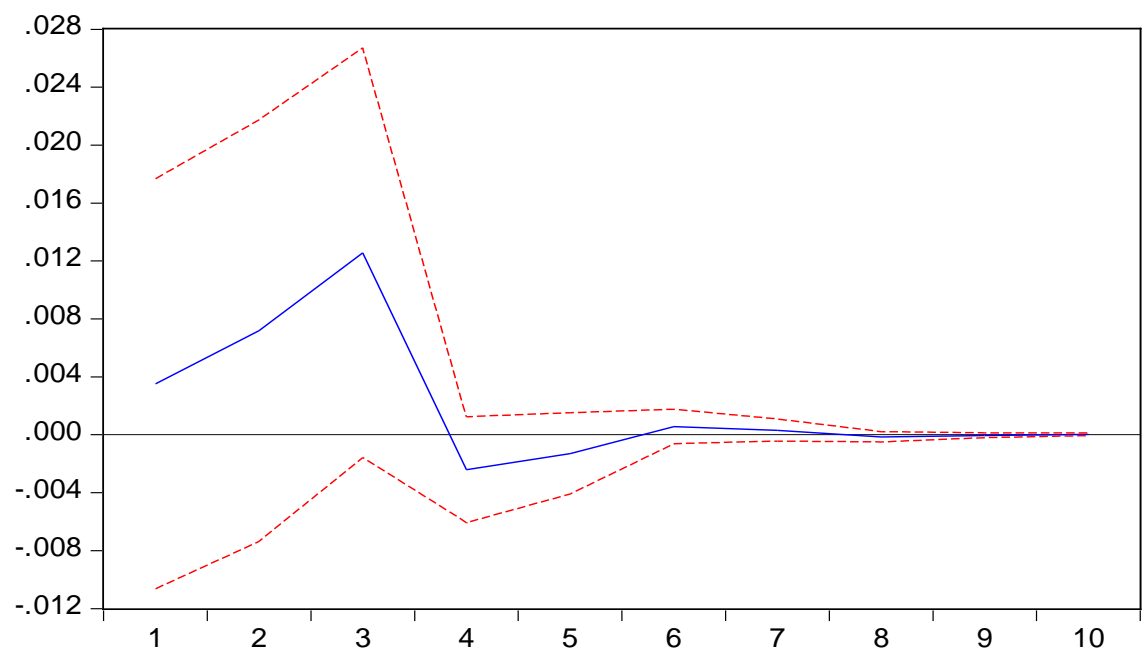

Figure 1. Impulse response of real estate price growth rate to higher education resource allocation growth rate.

range of the pulse effect fluctuation, that is, the actual impulse response changes between the upper and lower red lines. We can find from the figure that the amplitude range of the impulse response is small, but the response result is positive. The impulse response reaches the maximum value in the second and third periods, and gradually approaches zero as time passes. The information is that when we invest in the growth rate of higher education resource allocation in the first year, the growth rate of real estate prices has just begun to be positive, and the fluctuation has been small. In the fourth year, the impact on real estate prices began to diminish until it was zero.

Showing in Figure 2, when making an impulse response model, we can perform a root test on the impulse response result. The result is shown in Figure 2. From the figure, we can see that the eigenvalues fall within the unit circle, which means that the model is stable and can be very good. Explain the relationship between the two.

In the impulse response function, we can see that the growth rate of real estate prices has a lag to the growth rate of higher education resource allocation, which means that the dependent variable in the above formula may sometimes have strong autocorrelation. The dependent variable lag term is added to the equation to obtain a dynamic panel measurement model 3.

$$
\Delta \ln \mathrm{REP}_{i t}=\alpha_{i}+\sum_{j=1}^{p} \rho_{j} \Delta \ln \mathrm{REP}_{i t-j}+\beta_{1} \Delta \ln \mathrm{GDP}_{i t}+\varphi \Delta \ln \mathrm{AERI}_{i t}+\varepsilon_{i t}
$$




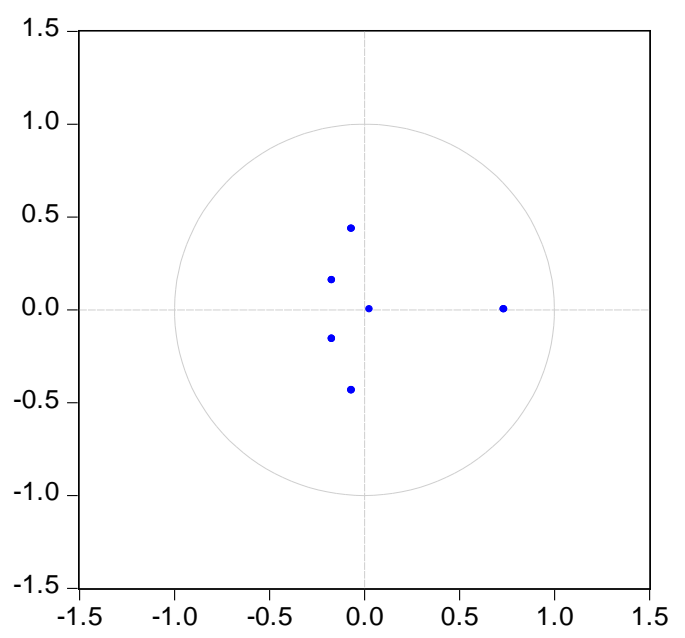

Figure 2. AR root test.

In this formula, $\Delta \ln \mathrm{REP}_{i t-j}$ is the real estate price growth rate after the correction.

In order to further quantify the previous relationship between the two, we must first determine the value in the dynamic panel model, that is, the optimal lag order. After specific operations, where the AIC index reaches the minimum value at the lag first order, the model The optimal lag order is 1 , and the measurement model can determine the form of the final measurement model 3 , which can be expressed as:

$$
\Delta \ln \mathrm{REP}_{i t}=\alpha_{i}+\rho_{1} \Delta \ln \mathrm{REP}_{i t-1}+\beta_{1} \Delta \ln \mathrm{GDP}_{i t}+\varphi \Delta \ln \mathrm{AERI}_{i t}+\varepsilon_{i t}
$$

\subsection{Empirical Analysis of Interaction}

The mechanism of the allocation of higher education resources and real estate prices is obtained from the impulse response model. Above, we are empirically analyzing the interaction between the two based on dynamic panel data models. The interactive relationship between them, panel data regression on the allocation of higher education resources and real estate prices, the results are shown in Table 3.

Showing in Table 3, for regression analysis of econometric model 1, we find that at a significant level of $1 \%$, the coefficient of urban GDP growth rate is significantly positive, which means that GDP growth rate has a positive effect on the growth rate of real estate prices, and the regression coefficient is 0.3988 . In other words, for every $1 \%$ increase in GDP growth rate of a city, the growth rate of real estate prices will increase by $0.3988 \%$.

The variable of growth rate of higher education resource allocation is introduced in Model 2. According to the regression results, we can find that without the introduction of a lag term, the growth rate of higher education resource allocation is not significant in the regression result of the growth rate of real estate prices, that is, higher in this model. The growth rate of educational resources is not relevant. 
Table 3. Empirical test results.

\begin{tabular}{cccc}
\hline & Model 1 & Model 2 & Model 3 \\
\hline C & $0.0456^{* * *}$ & $0.04297^{* * *}$ & $0.0521^{* * *}$ \\
& $(2.9077)$ & $(2.7175)$ & $(3.2864)$ \\
$\Delta \operatorname{lnGDP}$ & $0.3988^{* * *}$ & $0.3709^{* * *}$ & $0.3627^{* * *}$ \\
& $(3.7267)$ & $(3.3938)$ & $(2.9217)$ \\
$\Delta \ln$ AERI & & 0.0670 & $0.0037^{* *}$ \\
& & $(1.2282)$ & $(2.2640)$ \\
$\Delta \ln R E P(-1)$ & & $-0.1123^{* *}$ \\
Adj-R2 & 0.0397 & & $(-1.7710)$ \\
F-statistic & $13.8880^{* * *}$ & 0.0412 & 0.0215 \\
\hline
\end{tabular}

Through the impulse response function, we found that the model variable has a lag, so the lag term is added to the model 2 to obtain the measurement model 3. According to the regression results of econometric model 3 , we find that at a significant level of $5 \%$, the coefficient of the growth rate of higher education resource allocation is significantly positive, which means that the growth rate of higher education resource allocation has a positive effect on the growth rate of real estate prices. Consistent with our previous analysis.

\section{Disadvantages and Shortcomings}

The shortcomings and deficiencies of this paper are mainly reflected in the following two aspects. On the one hand, the effect of real estate prices on the allocation of higher education resources is not explored; the endogenous nature of the econometric model is not effectively addressed; Taking an evaluation system for measurement will have a certain degree of subjectivity, which will cause a small deviation between the empirical results and the actual results.

\section{Conclusion and Policy Recommendations}

By studying the interactive relationship between higher education resource allocation and real estate prices, theoretically studying domestic and foreign literature, qualitative analysis of higher education resources, higher education resource allocation and other related concepts and the connotation of some related methods, thus providing a systematic Research ideas and methods; on the one hand, based on the relevant conclusions of theoretical analysis, combined with the current situation of higher education resource allocation, a reasonable evaluation system for higher education resource allocation is constructed, and a comprehensive index of higher education resource allocation is obtained through principal component analysis to discover higher education resource allocation. Most of the higher degrees are large cities in the first and second tiers, while the allocation of higher education resources in small and medium cities is low.

On the other hand, a basic econometric model is established. By introducing 
variables of higher education resources into the basic model and establishing an impulse response model, it is found that there is a lag effect. The first-order lag term is added to obtain the final econometric model. Through regression analysis, we found that under the significance of $5 \%$, the regression results show that the growth rate of higher education resource allocation has a positive impact on the growth rate of real estate prices. From the perspective of economics, we believe that the speed of higher education resource allocation in a city is accelerating. The attractiveness for full-time teachers and college students for registration increases, and the potential demand of full-time teachers and college students for the real estate market will stimulate the acceleration of the growth rate of real estate prices.

There are various fluctuations in real estate prices in various cities in China. The rapid growth of housing prices in first- and second-tier cities has become a difficult social problem. Nowadays, various cities in China have adopted various measures to regulate the real estate market. Such measures, combined with the actual results, it is still difficult to solve the excessive growth of real estate prices from the perspective of purchase restrictions and other regulatory policies. Based on the empirical analysis of this article, we may start with the allocation of higher education resources. The allocation of higher education resources is centralized, and the government can take effective measures to allocate higher education resources to avoid excessive regional differences in the allocation of higher education resources, which can slow the social pressure brought by the increase in real estate prices to a certain extent.

\section{Acknowledgements}

The successful completion of the thesis is grateful to the teachers and classmates who have given me guidance. From the topic selection to the finalization of the dissertation, I have your help in all aspects, so that I can master and use professional knowledge and conduct in-depth research and analysis of the thesis. As time goes by, I also thank my classmates for their encouragement and support during the three years of development, spend the most beautiful time together, make college life so memorable, and wish everyone a dream come true!

\section{Conflicts of Interest}

The author declares no conflicts of interest regarding the publication of this paper.

\section{References}

Bao, W., \& Liu, Y. H. (2011). An HLM Analysis of Disparities in the Resources Alloction of China's Higher Education. Research in Educational Development, 19.

Chen, L. N., \& Wang, H. (2012). Empirical Investigation on the Regional Interactions of Real Estate Prices in China. Statistical Research, 29.

Du, X. J., Huang, Z. H., \& Wu, C. F. (2009). Study on Interaction among Housing Price, Public Expenditure and Real Estate Taxes. The Journal of Quantitative \& Technical 
Economics, No.1, 109-119.

$\mathrm{Hu}, \mathrm{X}$. (2010). Income Inequality and China's Real Estate Prices: Theoretical and Empirical Analysis. Finance \& Economics, No. 3, 86-96.

Li, F., \& Li, Q. J. (2014). Interactive Relationship between RMB Exchange Rate and Real Estate Prices. Studies of International Finance, No.12, 83-90.

Zhang, H., Li, Z. F., \& Deng, B. J. (2014). Educational Resource Allocation Mechanisms and Housing Prices: An Empirical Analysis of Education Capitalization in China. Journal of Financial Research, No.5, 193-206.

Zhu, Y. Z., \& Xu, D. (2013). Official Promotion Pressures, Financial Marketization and House Price Growth. Journal of Financial Research, No. 1, 65-78. 